\title{
Capacidad de desarrollo embrionario de ovocitos de bovino recuperados vía ultrasonografía y de ovarios de matadero
}

\author{
Embryonic development capacity of bovine oocytes aspired by ovum pick-up \\ and from slaughterhouse ovaries \\ Carlos Quispe E. ${ }^{1,4}$, Edith Ancco G. ${ }^{1}$, Juan Solano A. ${ }^{2}$, Ide Unchupaico P. ${ }^{3}$, Edwin \\ Mellisho S. ${ }^{1}$
}

\section{Resumen}

\begin{abstract}
El objetivo del presente estudio fue comparar la capacidad de desarrollo embrionario de ovocitos recuperados de ovarios de matadero y recuperados in vivo mediante ultrasonografía guiada (ovum pick-up, OPU) en un sistema de producción in vitro. Se realizaron nueve sesiones de colecta en 59 vacas Holstein para la obtención de ovocitos mediante OPU y se colectaron 408 ovarios de 204 vacas de matadero. Para la maduración in vitro de ovocitos solo se utilizaron aquellos de grado 1 y 2 mediante medios MIV, FIV y CIV ${ }^{\circledR}$ hasta el día 7. Se evaluó la tasa de recuperación de ovocitos, ovocitos grado 1 y 2 y el porcentaje de embriones (blastocitos) producidos in vitro al día 7. La tasa de recuperación de ovocitos fue mayor cuando se colectaron de ovarios de matadero comparado con ovocitos colectados por OPU ( 10.0 vs. 7.2 ovocitos por sesión/vaca; $p<0.05$ ). Hubo una mayor cantidad de ovocitos viables de grado 1 y 2 cuando la colecta fue de ovarios de matadero ( 7.6 vs. 3.4 ovocitos por sesión/vaca; $\mathrm{p}<0.05$ ). La tasa de blastocitos fue de 30.5 y $20.8 \%$ con ovocitos de ovarios de matadero y por OPU, respectivamente $(\mathrm{p}<0.05)$. Se obtuvo una mayor capacidad de desarrollo de ovocitos recuperados de ovarios de matadero que de aquellos recuperados por ovum pick-up.
\end{abstract}

Palabras clave: ovocitos; blastocitos; desarrollo embrionario; ovum pick-up

\footnotetext{
${ }^{1}$ Laboratorio de Biotecnología Reproductiva, Facultad de Zootecnia, Universidad Nacional Agraria La Molina, Lima, Perú

${ }^{2}$ Escuela Profesional de Medicina Veterinaria y Zootecnia, Universidad Peruana Los Andes, Junín, Perú

${ }^{3}$ Facultad de Zootecnia, Universidad Nacional del Centro del Perú, Junín, Perú

${ }^{4}$ E-mail: cquispe.vet@gmail.com
} 


\begin{abstract}
The aim of this study was to compare the capacity of embryonic development of oocytes recovered from slaughterhouse ovaries and recovered in vivo by guided ultrasound (ovum pick-up - OPU) in an in vitro production system. Nine sessions in 59 Holstein cows were done to obtain oocytes through OPU and 408 ovaries were collected from 204 cows in the slaughterhouse. For the in vitro maturation of oocytes, only those of grade 1 and 2 were used, matured and fertilized using MIV, FIV and CIV ${ }^{\circledR}$ medium until day 7. The recovery rate of oocytes, grade 1 and 2 oocytes and the percentage of embryos (blastocysts) produced in vitro on day 7 were evaluated. The recovery rate of oocytes collected from slaughterhouse ovaries was higher as compared with oocytes collected by OPU ( 10.0 vs. 7.2 oocytes per session/cow, $p<0.05)$. There was a greater number of viable oocytes of grade 1 and 2 when the collection was from slaughterhouse ovaries ( 7.6 vs. 3.4 oocytes per session/cow, $\mathrm{p}<0.05$ ). The blastocyst rate was 30.5 and $20.8 \%$ with slaughterhouse ovarian oocytes and OPU, respectively $(\mathrm{p}<0.05)$. A greater capacity to develop oocytes retrieved from slaughterhouse ovaries was obtained than those recovered by ovum pick-up.
\end{abstract}

Key words: oocytes; blastocysts; embryonic development; ovum pick up

\section{INTRODUCCIÓN}

La producción de embriones in vitro es una biotecnología desarrollada para obtener crías de de animales de producción de alto valor genético (Palmer et al., 1991). La técnica en su conjunto implica la maduración in vitro de ovocitos (MIV), fecundación in vitro de ovocitos madurados (FIV) y el cultivo in vitro de ovocitos fertilizados (CIV) hasta el estado de blastocisto en el día 7 en el caso del bovino. La aplicación de la fecundación in vitro se puede realizar en vacas vacías, vacas gestantes, vaquillas e incluso en donantes con problemas de fertilidad o que la función reproductiva se encuentra mermada frente a los tratamientos de superovulación y de sincronización de celo (Anchordoquy et al., 2013). Los ovocitos pueden ser obtenidos a partir de ovarios de vacas beneficiadas y donantes vivos, así como de hembras con diversos antecedentes de ciclos reproductivos (Bilodeau-Goeseels y Panich, 2002). Para la recuperación de ovocitos de animales vivos generalmente se utiliza la técnica de aspiración folicular guiada por ultrasonografía
(OPU), que permite incrementar el número de crías de donantes genéticamente valiosas (Galli et al., 2001).

La condición fisiológica del animal indica que solamente el $0.1 \%$ de los folículos alcanzan la ovulación (Gosden y Telfer, 1987). Silva-Santos et al. (2011) mencionan que la población de folículos pre-antrales varía entre 89000 y 109000 en la transición de vaquilla a vaca, número que se reduce a 3000 folículos en vacas con más de 10 años (Erickson, 1996). La recuperación de ovarios de matadero como fuente de germoplasma con fines de fecundación in vitro permite obtener crías viables, aun cuando la donante ha sido beneficiada. Por otro lado, la punción de los folículos ováricos con la técnica de OPU (Ovum Pick-up) fue descrita por Pieterse et al. (1988) y es una técnica que permite incrementar los parámetros reproductivos con la producción eficiente de embriones in vitro. En ambos procedimientos es necesario conocer el potencial de recuperación de ovocitos y si estos adquieren una capacidad de desarrollo dentro de ciertos estándares bajo un sistema in vitro, ini- 
ciado con la evaluación del complejo ovocitocumulus (COCs).

La calidad de los ovocitos se define como la capacidad para producir un blastocisto dentro de un sistema de producción in vitro (Merton et al., 2003). Trabajos de investigación sugieren que la calidad propia de los ovocitos es el factor determinante para la capacidad de desarrollo al estadio de blastocisto (Lonergan et al., 2003), y este se relaciona con el estado fisiológico y reproductivo de los donantes, el tamaño del folículo, la extensión y la integridad de las células del cumulus (Lonergan et al., 1994; Liebfried-Rutledge, 1996).

Algunos reportes indican que los ovocitos recuperados de matadero son de mejor calidad y mayor cantidad en comparación a los recuperados por OPU (Critser et al, 1986; Mullaart et al., 1999), lo cual podría afectar la producción in vitro de embriones (Merton et al., 2003). El objetivo de esta investigación fue comparar la capacidad de desarrollo embrionario de ovocitos recuperados de ovarios de matadero y por OPU en un sistema de producción de embriones in vitro.

\section{Materiales y Métodos}

El estudio fue realizado en la Unidad Experimental de Zootecnia y en el Laboratorio de Biotecnología Reproductiva de la Facultad de Zootecnia de la Universidad Nacional Agraria La Molina (Lima, Perú) entre los meses de mayo y junio. La colección de ovocitos guiada por ultrasonografía fue realizada en 59 vacas Holstein divididas en nueve sesiones de colecta. Así mismo, se colectaron los ovarios de 204 vacas en el matadero. La diferencia entre los grupos fue estimada mediante una prueba de Chi cuadrado a un nivel de significancia de 0.05 . El paquete estadístico que se utilizó fue el SAS v. 9.0 y SPSS v. 15.0.

\section{Obtención del Complejo Ovocito- Cumulus (COCs)}

Aspiración de ovocitos de ovarios de matadero

Los ovarios se obtuvieron de un matadero local y se transportaron al laboratorio en solución isotónica temperada a $36 \mathrm{C}^{\circ}$ dentro de las tres horas del beneficio. Se utilizaron vacas de razas lecheras. Se aspiraron folículos de 2 a $8 \mathrm{~mm}$ de diámetro utilizando agujas N. ${ }^{\circ} 18$ y jeringa de $3 \mathrm{ml}$. El aspirado folicular fue vertido del tubo de colecta a una placa Petri temperada a $36^{\circ} \mathrm{C}$ en platina caliente. El medio utilizado para el conteo y clasificación de los ovocitos fue PBS (tampón fosfato salino) $+1 \%$ SFB (suero fetal bovino). Los ovocitos fueron contados en un estereoscopio Boeco ${ }^{\circledR}$ BS-80 y clasificados en cuatro grados de acuerdo a la apariencia de las células del cumulus que rodean el citoplasma (Liebfried y First, 1979; Sato et al., 1990) donde Grado 1: ovocitos completamente rodeados con más de tres capas de células del cumulus y citoplasma uniformemente granulada; Grado 2: ovocitos de una a tres capas de células y con citoplasma uniformemente granulada; Grado 3: ovocitos parcialmente desnudos con citoplasma desigual granulada; Grado 4: ovocitos completamente desnudos, degenerados. Solo los ovocitos de grado 1 y 2 dos fueron sometidos a MIV, mientras que los grados 3 y 4 fueron descartados.

Aspiración de ovocitos por Ovum Pick up $(O P U)$

Se realizó en vacas sin estimulación hormonal en nueve sesiones. Las 59 donantes estuvieron en estado sanitario óptimo y con edades entre 4 y 6 años. El día 1 se realizó una ecografía de inspección ovárica haciendo la ablación folicular manual en casos de hallarse folículos dominantes. El día 4 se realizó la OPU de acuerdo con el procedimiento descrito por Pieterse et al. (1988) y 
Getz (2004), aplicando anestesia epidural (4 $\mathrm{ml}$ de lidocaína $2 \%$ ) antes de la aspiración transvaginal de ovocitos. La aspiración folicular duró aproximadamente 10 minutos por cada vaca. La temperatura ambiental promedio fue de $20^{\circ} \mathrm{C}$.

Se utilizó un ecógrafo Mindray y transductor transvaginal de $7.5 \mathrm{MHz}$. Se utilizó una aguja de punción $21 \mathrm{G}$, que se adjunta a la guía de aspiración folicular (WTA ${ }^{\circledR}$, Brasil) a través de la vagina. Todos los folículos visibles $(>2 \mathrm{~mm}$ ) se perforaron y los contenidos fueron recogidos en un tubo estéril de 50 $\mathrm{ml}\left(\right.$ Falcon $\left.{ }^{\mathrm{TM}}\right)$ temperado a $36^{\circ} \mathrm{C}$ con medio de aspiración (PBS suplementado con heparina $0.02 \mathrm{mg} / \mathrm{ml}+1 \% \mathrm{SFB}$ ) unidos a una bomba de vacío para asegurar una presión de aspiración constante de $40 \mathrm{~mm}$ de $\mathrm{Hg}(\mathrm{MHz})$. El fluido folicular obtenido se llevó al laboratorio en el tiempo más breve posible. Los ovocitos recuperados fueron observados en un microscopio estereoscópico, se contaron y se clasificaron morfológicamente en cuatro categorías como se indicó anteriormente.

\section{Producción de Embriones in vitro}

\section{Maduración in vitro (MIV)}

Los ovocitos seleccionados fueron colocados individualmente en placas Petri, se lavaron 2-3 veces en medio H-199 (Hepes). Luego los ovocitos se lavaron 2-3 veces en gotas de $100 \mu 1$ en placas Petri con medio MIV (Vitrogen, Brasil) y se transfirieron a placas Petri de $35 \times 10 \mathrm{~mm}$, con gotas de maduración de $70 \mu \mathrm{l}$ cubiertas con aceite mineral, previamente equilibrado en la incubadora. Las placas Petri fueron preparadas con las gotas de maduración de $70 \mu \mathrm{l}$ y 10 ovocitos por gota, y se colocaron en la incubadora a 38.5 ${ }^{\circ} \mathrm{C}, 5 \%$ de $\mathrm{CO}_{2}$ y $90 \%$ humedad por $22-24 \mathrm{~h}$.

\section{Fecundación in vitro (FIV)}

Previa a la fecundación, los ovocitos fueron lavados en medio de fertilización FIV (Vitrogen, Brasil) 2-3 veces y se dejaron en gotas con medio de fertilización en la incubadora $\left(38.5^{\circ} \mathrm{C}\right.$ y $\left.5 \% \mathrm{CO}_{2}\right)$.

Se utilizó semen nacional de un toro Holstein. Las pajillas de semen fueron descongeladas a $37^{\circ} \mathrm{C}$ por $15 \mathrm{~s}$ y la motilidad fue evaluada con ayuda del equipo Computer Assisted Sperm Analysis (CASA) ISASv1 ${ }^{\circledR}$ con microscopio de contraste de fase UB203i. Luego se realizó la capacitación y selección espermática en gradientes de densidad (Percoll), colocándose $500 \mu 1$ de Percoll 45 en el gradiente superior (rosado) y $500 \mu 1$ de Percoll 90 en el gradiente inferior (transparente) y $200 \mu 1$ de semen. Se llevó a una primera centrifugación a $500 \mathrm{~g}$ por 15 min, se eliminó el sobrenadante, y luego se diluyó con $500 \mu 1$ de medio de fertilización (FIV). Se volvió a centrifugar a $500 \mathrm{~g}$ por 5 min y se eliminó el sobrenadante. Se determinó la concentración espermática y se colocaron 10000 espermatozoides motiles por ovocito, consistiendo en 4-5 $\mu 1$ de semen por cada gota de $70 \mu \mathrm{l}$ de medio FIV. Por último, se llevó a incubar por $18 \mathrm{~h}$ a $38.5^{\circ} \mathrm{C}, 5 \%$ de $\mathrm{CO}_{2}$ y $90 \%$ humedad.

\section{Cultivo in vitro (CIV)}

Culminada la etapa de fecundación, se removió el cumulus de los ovocitos hasta casi desnudarlos por completo. Se lavaron en medio de cultivo CIV (Vitrogen, Brasil), se colocaron en gotas de $70 \mu \mathrm{l}$ con 10 ovocitos por gota en placas Petri cubiertas con aceite mineral y se llevaron a la incubadora $38.5^{\circ} \mathrm{C}$ con $5 \% \mathrm{CO}_{2}$ y $90 \%$ de humedad. A las $48 \mathrm{~h}$ se realizó la primera renovación del $50 \%$ de medio CIV y se procedió a la evaluación de división celular. Luego se retiró el restante de las células del cumulus. En el día 5 se realizó la segunda renovación del 50\% del medio CIV. El día 7 se obtuvieron los embriones en el estado de blastocisto. La evaluación de los embriones se realizó bajo un microscopio estereoscópico utilizando los criterios morfológicos de la Sociedad Internacional de Transferencia de Embriones (IETS) (Wright, 1998). 
Cuadro 1. Efecto del origen de la colecta de ovocitos sobre la capacidad de desarrollo embrionario

\begin{tabular}{lccccc}
\hline \multirow{2}{*}{$\begin{array}{l}\text { Fuente de } \\
\text { ovocitos }\end{array}$} & $\begin{array}{c}\text { Animales } \\
(\mathrm{n})\end{array}$ & $\begin{array}{c}\text { Ovocitos } \\
\text { recuperados } \\
(\mathrm{n})\end{array}$ & $\begin{array}{c}\text { COC viables } \\
(\mathrm{n})\end{array}$ & \multicolumn{2}{c}{\begin{tabular}{c} 
Blastocistos (día 7) \\
\cline { 5 - 6 }$(\mathrm{n})$
\end{tabular}} \\
\hline $\begin{array}{l}\text { Vacas } \\
\text { donadoras }\end{array}$ & 59 & $7.0 \pm 2.1^{\mathrm{b}}$ & $3.4 \pm 1.5^{\mathrm{b}}$ & 66 & $\mathrm{n}$ \\
$\begin{array}{l}\text { Ovarios de } \\
\text { matadero }\end{array}$ & 204 & $10.0 \pm 2.1^{\mathrm{a}}$ & $7.6 \pm 2.7^{\mathrm{a}}$ & 374 & $30.5 \pm 20.7^{\mathrm{b}}$ \\
\hline
\end{tabular}

Letras diferentes dentro de cada columna indican diferencia significativa entre grupos $(p<0.05)$

${ }^{1}$ Ovocitos grado 1 y 2

\section{Resultados}

Se obtuvieron un total de 2049 ovocitos en 18 repeticiones de colecta provenientes de 408 ovarios de 204 vacas beneficiadas (10.0 ovocitos por hembra). En el caso de la aspiración de ovocitos por ovum pick-up, se colectaron 414 ovocitos de 59 vacas en 9 sesiones (7.1 ovocitos por vaca), siendo estadísticamente superior el número de ovocitos colectados por vaca en el matadero $(\mathrm{p}<0.05)$. Así mismo, hubo una mayor cantidad de ovocitos viables de grado 1 y 2 cuando la colecta fue de ovarios de matadero que por OPU (7.6 vs. 3.4 ovocitos por vaca, respectivamente). La tasa de blastocitos al día 7 fue 30.5 y $20.8 \%$ provenientes de ovarios de matadero y por OPU, respectivamente $(p<0.05)$. Los datos se presentan en el Cuadro 1.

\section{Discusión}

La cantidad de ovocitos recolectados por aspiración transvaginal de 7.1 por vaca/ sesión fue ligeramente superior a lo encontrado por Karadjole et al. (2010) y Getz (2004). Diversos factores pueden influenciar el número de ovocitos recolectados, tales como los tratamientos hormonales, el núme- ro de aguja de punción, la frecuencia de la aspiración, la presión de vacío, la resolución del equipo utilizado, el tamaño de los folículos y la raza (Merton et al., 2003; Quispe et al., 2015). Bajo este contexto es que solo se recolectaron ovocitos por OPU cuyos folículos eran iguales o mayores de $2 \mathrm{~mm}$. Folículos menores a $2 \mathrm{~mm}$ son difíciles de ver en el ecógrafo (Quispe et al., 2014). Por el contrario, folículos de $1 \mathrm{~mm}$ pueden ser visibles en los ovarios de matadero, lo que contribuye a la mayor cantidad de folículos perforados (Getz et al., 2004; Karadjole et al., 2007; Merton et al., 2003). Además, el número promedio de ovocitos colectados por OPU en ganado Bos taurus es menor a ocho por donante (Senatore et al., 2010).

Se reconoce un mayor porcentaje de embriones producidos de ovocitos colectados de ovarios de matadero debido a que estos están exentos de factores que limitan su calidad como la presión de vacío y el diámetro de la aguja utilizados durante la aspiración (Hashimoto et al., 1999). Los COCs están menos adheridos a los folículos en ovarios de matadero debido al efecto post mortem, de allí que tienen cúmulos más completos (Mullaart et al., 1999; Karadjole et al., 2010). Los laboratorios de FIV procuran madurar la mayor cantidad de ovocitos procedentes de una aspiración in vivo a fin de aprovechar al 
máximo la producción de embriones, y en muchos casos ovocitos de grado 3 logran alcanzar el estado de blastocistos con cierta calidad (Watanabe, 2001). Esto puede contribuir a mejores rendimientos de blastocistos obtenidos de ovocitos derivados de mataderos.

Los resultados del presente estudio muestran 3.4 ovocitos viables de grado 1 y 2 cuando la colecta es realizada por OPU, inferior a lo reportado por Anchordoquy et al. (2013) con un promedio de 4.3 ovocitos viables en Bos taurus. Así mismo, la tasa de blastocistos de ovocitos derivados de OPU fue menor que la de los ovocitos aspirados de ovarios de matadero. No obstante, Karadjole et al. (2010) reportan una mayor tasa de embriones producidos con ovocitos derivados de OPU que con ovocitos de matadero. Por otro lado, el tamaño de los folículos puede explicar las diferencias en la tasa de blastocistos debido a que la mayoría de los ovocitos recogidos de ovarios de matadero en el presente estudio se obtuvo de folículos entre 1 y $4 \mathrm{~mm}$ (Blondin y Sirard, 1995; Karadjole, 2009).

\section{Conclusiones}

Los resultados demuestran que los ovocitos recuperados de ovarios de bovinos de matadero tienen mayor capacidad de desarrollo que los colectados in vivo mediante la técnica aspiración guiada por ultrasonografía (ovum pick-up) para alcanzar el estado de blastocisto.

\section{Literatura Citada}

1. Anchordoquy JP, Huter S, Farnetano N, Iroulegu Y, Martin E, Arzeno M, Mijica I, et al. 2013. Producción de embriones por fecundación in vitro de donantes problema de razas taurinas. Spermova 3: 126-129.
2. Bilodeau-Goeseels S, Panich P. 2002. Effects of oocyte quality on development and transcriptional activity in early bovine embryos. Anim Reprod Sci 71: 143-155. doi: 10.1016/S0378-4320(01)00188-9

3. Blondin P, Sirard MA. 1995. Oocyte and follicular morphology as determining characteristics for developmental competence in bovine oocytes. Mol Reprod Dev 41: 54-62. doi: 10.1002/ mrd.1080410109

4. Critser ES, Leibfried-Rutledge ML, Eyestone WH, Northey DL, First $N L$. 1986. Acquisition of developmental competence during maturation in vitro. Theriogenology 25: 150. doi: 10.1016/ 0093-691X(86)90204-9

5. Erickson BH. 1996. Development and senescence of the bovine ovary. J Anim Sci 25: 800-805.

6. Galli C, Crotti G, Notari C, Turini P, Duchi R, Lazzari G. 2001. Embryo production by ovum pick up from live donors. Theriogenology 55: 1341-1357. doi: 10.1016/S0093-691X(01)00486-1

7. Getz I. 2004. The efficacy of ovarian stimulation in donor cows on the results of ovum pick up and in vitro embryo production. PhD Thesis. Croatia: University of Zagreb.

8. Gosden RG Telfer E. 1987. Numbers of follicles in mammalian ovaries and their allometric relationships. J Zool 211: 169-175. doi: 10.1111/j.1469-7998.1987.tb07460.x

9. Hashimoto S, Takakura R, Kishi M, Sudo T, Minami N, Yamada M. 1999. Ultrasound-guided follicle aspiration: the collection of bovine cumulus-oocyte complexes from ovaries of slaughtered or live cows. Theriogenology 51: 757-765. doi: 10.1016/S0093-691X(99)00024-2

10. Karadjole M. 2009. The effect of follicle size on oocyte quality and embryo development after in vitro fertilization PhD Thesis. Croatia: University of Zagreb. 
11. Karadjole M, Getz I, Samardžija M, Matkoviae M, Makek Z, Karadjole T, Baèia G, et al. 2007. The effect of origin of the immature oocytes on in vitro developmental competence of bovine embryos. Proc $23^{\text {th }}$ Annual Meeting of European Embryo Transfer Association. Alghero, Italy.

12. Karadjole M, Getz I, Samardžija M, Maceešice N, Matkoviae M, Makek Z, Karadjole T, et al. 2010. The developmental competence of bovine immature oocytes and quality of embryos derived from slaughterhouse ovaries or live donors by ovum pick up. Vet Arhiv 80: 445-454.

13. Liebfried L, First NL. 1979. Characterization of bovine follicular oocytes and their ability to mature in vitro. J Anim Sci 48: 76-86. doi: 10.2527/ jas1979.48176x

14. Liebfried-Rutledge ML. 1996. Factors determining competence of in vitro produced cattle embryos. Theriogenology 51: 473-485. doi: 10.1016/S0093691X(98)00241-6

15. Lonergan P, Rizos D, Fair T, Boland MP. 2003. In vitro production of bovine embryos: factors affecting blastocyst yield and quality. Proc IV MiddleEuropean Buiatric Congress. Lovran, Croatia.

16. Lonergan P, Monaghan P, Rizos D, Boland MP, Gordon I. 1994. Effect of follicle size on bovine oocyte quality and developmental competence following maturation, fertilization and culture in vitro. Mol Reprod Dev 37: 48-53. doi: 10.1002/mrd.1080370107

17. Merton JS, de Roos AP, Mullaart E, de Ruigh L, Kaal L, Vos PL, Dieleman SJ. 2003. Factors affecting oocyte quality and quantity in commercial application of embryo technologies in the cattle breeding industry. Theriogenology 59: 651-674. doi: 10.1016/S0093691X(02)01246-3
18. Mullaart E, Verbrugge A, Aerts B, Merton JS. 1999. Optimization of OPU procedure. Proc XV Scientific Meeting of European Embryo Transfer Association. Lyon, France.

19. Palmer EL, Bézard J, Magistrini M, Duchamp G. 1991. In vitro fertilization in the horse. A retrospective study. J Rep Fertil 44: 375-384.

20. Pieterse MC, Kappen KA, Kruip M, Taverne M. 1988. Aspiration of bovine oocytes during transvaginal ultrasound scanning of ovaries. Theriogenology 30 : 751-762. doi: 10.1016/0093-691X(88)90310-X

21. Quispe C, Mercado J, Fernández E, Mixan E, Gamarra S, Mellisho E. 2014. Efecto de tratamientos con benzoato de estradiol o GNRH sobre la dinámica folicular para aspiración de folículos (ovum pick up) guiada por ultrasonido en vacas lecheras. Spermova 4: 86-88.

22. Quispe C, Fernández E, Ancco E, Oriundo K, Mellisho E. 2015. Efecto de la raza de la donadora sobre la cantidad y calidad de ovocitos recuperados por aspiración folicular guiada por ultrasonografia transvaginal. Spermova 5: 59-62. doi: 10.18548/aspe/0002.13

23. Sato E, Matsuo M, Miyamoto $\boldsymbol{H}$. 1990. Meiotic maturation of bovine oocytes in vitro: improvement of meiotic competence by dibutyryl cyclic adenosine 3', 5'-monophosphate. J Anim Sci 68: 1182-1187. doi: 10.2527/1990.6841182x

24. Senatore EM, Xu J, Suárez MV, Gong G, Lin T, Bella A, Moreno JF, et al. 2010. Improved in vitro development of OPU-derived bovine (Bos taurus) embryos by group culture with agaroseembedded helper embryos. Theriogenology 74: 1643-1651. doi: 10.1016/j.theriogenology.2010.06.037

25. Silva-Santos K, Seneda M. 2011. Multioocyte follicles in adult mammalian ovaries. Anim Reprod 8: 58-67. 
26. Watanabe R. 2001. Aspiração in vivo de oócitos em fêmeas Nelore de diferentes idades reprodutivas mediante punção transvaginal guiada por ultrasom. Tese de Mestrado. Sao Paulo: Universidade Estadual Paulista. 52 p.
27. Wright JM. 1998. Photographic illustrations of embryo developmental stage and quality codes. In: Manual of the International Embryo Transfer Society. $3^{\text {rd }}$ Illinois, USA: IETS. p 167-170. 